\title{
Avaliação do grau de satisfação e qualidade de vida dos portadores de prótese dental
}

\author{
Assessment of the satisfaction level and quality of life of dental \\ prosthesis users
}

\author{
Wanessa Borges Beloni* \\ Hugo Felipe do Vale** \\ Jessica Mie Ferreira Koyama Takahashi**
}

\section{Resumo}

Diante da importância de oferecer um tratamento reabilitador adequado às condições dos pacientes atendidos em uma policlínica odontológica, busca-se saber quais as suas opiniões quanto à satisfação da instalação e utilização de próteses removíveis confeccionadas por estudantes de graduação em Odontologia. Objetivo: este estudo tem como objetivo a avaliação do grau de satisfação e qualidade de vida dos pacientes edentados totais e parciais reabilitados por estudantes de graduação. Materiais e método: trinta e dois pacientes foram chamados dois meses após a conclusão do tratamento reabilitador protético para consulta de proservação das próteses. Neste momento, os pacientes foram avaliados por meio do questionário OHIP-EDENT e por meio da escala visual analógica (EVA) para avaliar os critérios de qualidade de vida, estética, fonética, função mastigatória, estabilidade, conforto, alteração do paladar e dor referentes à prótese instalada. Os resultados foram submetidos ao teste $t$ com nível de significância de 5\%. Resultados: os pacientes reabilitados com prótese total apresentaram maior impacto na qualidade de vida $(p=0,01)$, relataram melhor estabilidade $(p=0,01)$ e função mastigatória $(p=0,01)$ em comparação aos reabilitados com próteses parciais. Não houve diferença nos critérios conforto, estética, fonética, alteração de paladar e dor ( $p>0,05)$. Conclusões: o tipo de prótese pode influenciar no prognóstico da reabilitação. Além disso, pacientes reabilitados com próteses totais relatam maior percepção de melhora na qualidade de vida após a reabilitação.

Palavras-chave: Prótese dentária. Satisfação do paciente. Qualidade de vida.

\section{Introdução}

Vários levantamentos epidemiológicos têm evidenciado a situação da saúde bucal no Brasil, demonstrando que são 30 milhões os indivíduos desdentados que necessitam de algum tipo de prótese dental ${ }^{1,2}$.

As próteses dentais são consideradas substitutos artificiais para os dentes que foram perdidos, devolvendo, além da função mastigatória, a estética e a fonética ao paciente ${ }^{3}$. Diante de uma situação de edentulismo, existem próteses dentais que substituem total ou parcialmente a quantidade de dentes ausentes, devendo a indicação do tipo de prótese ser precedida de anamnese, exame clínico e diagnóstico ${ }^{3}$.

Atualmente, o profissional tem várias opções para reabilitação oral de seus pacientes, incluindo próteses adesivas, parciais fixas, parciais removíveis e próteses totais aliadas ou não à utilização de implantes dentais. No entanto, o fator socioeconômico ainda é decisivo na busca do tratamento reabilitador oral ${ }^{4-7}$.

Seja qual for o tipo de prótese dental utilizada, a reabilitação oral influencia psicossocialmente a vida do paciente. É verificado que a ausência de dentes influencia diretamente o cotidiano das pessoas, afetando a sua qualidade de vida. Assim, mais que restabelecer a função mastigatória, ao procurar o tratamento reabilitador, os pacientes buscam reconstituir sua imagem pessoal, social e sua qualidade de vida ${ }^{8,9}$.

Graduanda em Odontologia - Escola Superior de Ciências da Saúde - Universidade do Estado do Amazonas, Manaus, Amazonas, Brasil.

Mestre, professor da área de Periodontia - Escola Superior de Ciências da Saúde - Universidade do Estado do Amazonas, Manaus, Amazonas, Brasil.

** Doutora, professora da área de Prótese Dental - Escola Superior de Ciências da Saúde - Universidade do Estado do Amazonas, Manaus, Amazonas, Brasil. 
A qualidade de vida é um termo generalista ou abrangente que remete ao bem-estar físico, psicológico e social de um indivíduo ${ }^{10}$. Apesar da ampla base científica na qual se apoia a Odontologia, existe uma lacuna a respeito do pensamento e sentimento do paciente em relação ao seu tratamento, sendo os critérios de sucesso estabelecidos pelo profissional, e não pelo paciente ${ }^{10,11}$. Diante disso, tem-se a importância de lançar mão de meios ou indicadores subjetivos para conhecer e mensurar a percepção do paciente em relação à sua saúde oral e, assim, poder identificar falhas, determinar a necessidade real de tratamento, complementar informações e medir os benefícios criados pelo tratamento protético ${ }^{12}$.

Dessa maneira, o objetivo deste estudo é avaliar o grau de satisfação e qualidade de vida dos pacientes edentados totais e parciais quando da utilização de próteses dentais.

\section{Materiais e método}

Este estudo foi realizado na Policlínica Odontológica da Escola Superior de Ciências da Saúde da Universidade do Estado do Amazonas (UEA), mediante aprovação do Comitê de Ética em Pesquisa desta universidade sob o protocolo $\mathrm{n}^{\circ} \mathrm{0}$ 054/12.

Foram selecionados, do banco de dados da instituição (amostra de conveniência), 39 pacientes adultos de ambos os gêneros (maiores de 18 anos) e reabilitados com prótese dental total removível (PT) ou prótese parcial removível (PPR) superior ou inferior. A seleção dos pacientes foi realizada com base nos seguintes critérios: inclusão - todos os pacientes reabilitados com prótese parcial removível ou prótese total confeccionada e instalada por acadêmico de graduação da Universidade do Estado do Amazonas no primeiro semestre de 2012; exclusão - pacientes impossibilitados de retornar à instituição por motivo de saúde ou indisponibilidade em comparecer à consulta. Dos 39 pacientes inicialmente selecionados, 32 participaram do estudo, por não apresentarem impedimento (indisponibilidade ou impossibilidade de retornar à instituição), assinando o termo de consentimento livre e esclarecido (TCLE).

Os pacientes foram chamados para consulta de proservação das próteses, após dois meses de uso, sendo novamente submetidos a anamnese, exame clínico e ajustes nas próteses, quando necessário. Ao final da consulta, responderam individualmente aos questionários Oral Health Impact Profile (OHIP-EDENT) $)^{13}$, para avaliar o impacto da condição oral na qualidade de vida, e escala visual analógica $(\mathrm{EVA})^{14}$, para avaliar o grau de satisfação com a reabilitação nos seguintes critérios: conforto, estabilidade/retenção, função mastigatória, estética, fonética, alteração do paladar e dor.
O OHIP-EDENT é um questionário específico para indivíduos edêntulos, apresentando, em sua versão brasileira, 19 perguntas distribuídas em sete domínios, e procura investigar: limitação funcional; dor física; desconforto psicológico; limitação física, psicológica, social e incapacidade devido a problemas com dentes, boca ou dentaduras ${ }^{13}$. Este instrumento subjetivo julga a percepção do indivíduo em relação à sua saúde bucal, levando em consideração expectativa na qualidade de vida ${ }^{15}$, sendo considerado adequado para avaliar os impactos no estilo de vida provindos da situação bucal em desdentados antes e/ou depois do tratamento ${ }^{16}$. Para cada pergunta do questionário foram apresentadas três opções de resposta (nunca, às vezes ou quase sempre). Após respondido o questionário, foram atribuídas pontuações de 0 (nunca), 1 (às vezes) ou 2 (sempre) para cada resposta. A somatória das pontuações de todas as perguntas gerou o índice OHIP e podia variar de 0 a 38. Quanto maior o índice OHIP, maior a percepção de impacto negativo da saúde bucal na qualidade de vida do indivíduo.

Dentre os vários métodos psicométricos existentes para mensurar a percepção e satisfação do indivíduo, utilizou-se, neste estudo, o questionário em EVA, no qual se apresentou um questionário composto de sete perguntas, havendo para cada pergunta uma escala de 0 a 10 . Nos extremos, 0 correspondeu a totalmente insatisfeito e 10, a completamente satisfeito. Foram considerados satisfeitos em suas habilidades nos diferentes domínios do questionário os entrevistados que assinalaram acima de sete na EVA.

Para a análise dos dados, dividiu-se a amostra em 2 grupos: pacientes reabilitados com prótese total e pacientes reabilitados com prótese parcial removível, sendo comparados dicotomicamente em cada um dos critérios avaliados. Os resultados obtidos foram submetidos a análise estatística (SPSS ${ }^{\circledR}$ Statistics Version 20 - IBM, Chicago, Illinois, EUA), utilizando o teste t não pareado com nível de significância de $5 \%$.

\section{Resultados}

Foram entrevistados 32 pacientes, sendo 24 do gênero feminino e oito do masculino. A faixa etária variou de 37 a 63 anos. Foram avaliados 17 pacientes reabilitados com prótese total superior, um com prótese total dupla, oito com prótese parcial removível superior e sete com prótese parcial removível inferior.

Os resultados obtidos nos questionários e na análise estatística estão relacionados na Tabela 1. 
Tabela 1 - Análise do grau de satisfação (OHIP) e qualidade de vida (EVA) - média e desvio padrão - de acordo com o tipo de prótese confeccionada

\begin{tabular}{c|l|c|c|c}
\hline \multirow{2}{*}{ Índice } & \multicolumn{1}{|c|}{ Critério } & \multicolumn{2}{|c|}{ Tipo de prótese } & \multirow{2}{*}{ Valor de $\mathrm{p}^{*}$} \\
\cline { 3 - 4 } OHIP & Qualidade de vida & PT & \multicolumn{1}{c}{ PPR } \\
\cline { 2 - 3 } & Conforto & $7,88(5,12)$ & $13,88(7,75)$ & 0,01 \\
& Estabilidade / Retenção & $9,25(1,34)$ & $8,31(1,89)$ & 0,12 \\
& Função mastigatória & $9,88(0,34)$ & $8,44(1,97)$ & 0,01 \\
\multirow{2}{*}{ EVA } & Estética & $9,12(1,36)$ & $7,25(2,46)$ & 0,01 \\
& Fonética & $9,69(0,70)$ & $9,62(1,26)$ & 0,86 \\
& Alteração do paladar & $9,62(0,72)$ & $9,00(1,41)$ & 0,13 \\
& Dor & $9,06(1,24)$ & $8,50(2,66)$ & 0,45 \\
\hline
\end{tabular}

* Se o valor de $\mathrm{p}>0,05$ não há diferença estatística entre os grupos de acordo com o teste t não pareado.

De acordo com o OHIP, os pacientes reabilitados com próteses dentais totais tiveram melhor percepção na qualidade de vida que os pacientes reabilitados com próteses parciais removíveis $(p=0,01)$. Os pacientes portadores de prótese total relataram, ainda, maior satisfação com relação à estabilidade $(\mathrm{p}=0,01)$ e função mastigatória $(\mathrm{p}=0,01)$ de suas próteses.

Não houve diferença quando comparados os quesitos conforto, estética, fonética, alteração de paladar e dor $(\mathrm{p}>0,05)$. A idade e o gênero também não influenciaram os resultados $(p>0,05)$.

\section{Discussão}

Os indicadores subjetivos têm sido utilizados na prática clínica como importante instrumento na reabilitação oral protética dos pacientes, na mensuração da percepção da sua saúde bucal, complementando informações da necessidade, bem como dos benefícios criados pelo tratamento reabilitador protético $^{12}$. Esses indicadores podem ser usados para complementar informações clínicas dos pacientes idosos $^{5}$, revelando sua expectativa com a própria saúde em vários aspectos e tendo o propósito maior de avaliar a sua qualidade de vida ${ }^{17}$.

O OHIP foi desenvolvido para avaliar o impacto social (bem-estar e qualidade de vida) causado pela condição de saúde bucal nos quesitos limitação funcional, dor física, desconforto psicológico, incapacidade física, psicológica e social e deficiência na realização das atividades cotidianas ${ }^{18,19}$.

De acordo com os resultados do OHIP, os pacientes reabilitados com próteses totais relataram melhor índice de qualidade de vida em comparação aos pacientes reabilitados com próteses parciais removíveis, indicando maior aceitação e adaptação às suas reabilitações.
Neste estudo, mais da metade dos pacientes reabilitados com próteses totais recebeu próteses totais superiores, o que pode ter contribuído para a sua melhor aceitação. Esse tipo de prótese apresenta uma superfície de área basal maior, a qual, quando associada às estruturas anatômicas favoráveis da cavidade oral maxilar, como o rebordo residual, a abóbada do palato e a área de selamento periférico, beneficia o prognóstico da reabilitação. Outros fatores também auxiliam na manutenção de uma retenção, estabilidade e adesão da prótese total aos tecidos de suporte, por exemplo, a pressão atmosférica, que é considerada uma força de retenção e pode ser capaz de manter a prótese em seu local quando aplicadas forças no sentido de deslocá-la. Há, também, a adesão que é proporcionada pela adaptação em íntimo contato da base da prótese com os tecidos de suporte, interpostos por película de saliva ${ }^{20}$.

A literatura demonstra que pacientes reabilitados com próteses superiores as consideram satisfatórias quanto à retenção e à estabilidade, ao passo que classificam as próteses totais inferiores como insatisfatórias quanto à retenção e ao conforto ${ }^{21}$. Os usuários de prótese total, em sua maioria, estão satisfeitos com suas próteses superiores e insatisfeitos com suas próteses inferiores ${ }^{22}$.

Por sua vez, as próteses dentais inferiores apresentam uma área basal menor em comparação às próteses dentais superiores. A região de suporte da mandibula é menor que a da maxila e apresenta estruturas anatômicas que podem dificultar no prognóstico da reabilitação. Adicionalmente, as próteses inferiores estão sujeitas à movimentação da língua, que pode contribuir para o seu deslocamento e a sua desestabilização, inviabilizando o seu uso sem o devido ajuste. Durante o presente estudo, os pacientes reabilitados com próteses inferiores necessitaram de maior intervenção para ajuste. 
Analisando as próteses parciais removíveis, constatou-se que o maior número era de inferiores. Dos 20 pacientes, 12 eram portadores de próteses inferiores classe I de Kennedy, alguns com presença apenas dos dentes anteriores inferiores remanescentes. Esse tipo de prótese com extremidade livre possui apoio dentomucossuportado. Entretanto, nos casos com poucos dentes remanescentes, o suporte da prótese é principalmente em mucosa, e a retenção por meio dos grampos pode ficar comprometida, influenciando no prognóstico da reabilitação protética.

Todos os pacientes entrevistados apresentaram autopercepção e impacto da saúde bucal na qualidade de vida bastante positivos, sugerindo sua satisfação com a reabilitação oferecida. Estudo prévio ${ }^{23}$ demonstrou que, antes de reabilitados com próteses dentais, os indivíduos desdentados classificavam sua saúde bucal como ruim, indicando o desconforto e descontentamento com a condição de edentulismo. Porém, esses mesmos indivíduos indicam alta satisfação global com a vida. A divergência entre os resultados apresentados pode ser decorrente do tipo de questionário aplicado, no qual não se avaliou especificamente o impacto da saúde bucal na qualidade de vida, mas a qualidade de vida como um todo.

Em estudo onde se avaliou a expectativa dos pacientes em face da reposição protética dos dentes, a preocupação mais frequente envolveu a qualidade da prótese, especialmente quanto à estabilidade e à adaptação da prótese. Sabe-se que o método de avaliação do estado de saúde bucal mais comumente empregado é a avaliação clínica e que a perda total de dentes tem impacto na vida das pessoas ${ }^{16}$, implicando em consequências negativas tanto para o aspecto psicológico quanto para o social. Torna-se necessário que os profissionais da área da saúde tomem para si a responsabilidade e sensibilidade em prover o tratamento reabilitador mais adequado possível a pacientes portadores de próteses dentais, sejam elas parciais ou totais removíveis ${ }^{4}$.

\section{Conclusão}

Conclui-se que o tipo de prótese pode influenciar parâmetros subjetivos do grau de satisfação do paciente com a reabilitação e da qualidade de vida após a reabilitação oral com próteses totais ou próteses parciais removíveis. Os pacientes reabilitados com próteses totais relatam melhor percepção da qualidade de vida e maior satisfação com a estabilidade e a função mastigatória após a reabilitação.

\section{Agradecimento}

Este trabalho foi realizado com auxílio do Programa de Apoio à Iniciação Científica (PAIC), promovido pela Fundação de Amparo à Pesquisa do Estado do Amazonas (FAPEAM).

\section{Abstract}

Given the importance of offering proper rehabilitation for patients assisted in a dental polyclinic we have sought their opinion regarding installation and use of removable dental prosthesis manufactured by Dentistry students. Objective: this study aimed to assess the satisfaction level and quality of life of partially or totally edentulous patients rehabilitated by graduate students. Materials and method: thirty-two patients were called two months after the completion of the rehabilitating prosthetic treatment for an appointment of prosthesis proservation. At this time the patients were assessed through the OHIP-EDENT questionnaire and visual analogue scale (VAS) to assess criteria for quality of life, esthetics, phonetics, masticatory function, stability, comfort, palate alteration, and pain associated with the prosthesis installed. The results were submitted to t test with 5\% significance level. Results: the patients rehabilitated with complete dentures presented higher impact on quality of life $(p=0.01)$, and reported better stability $(p=0.01)$ and masticatory function $(p=0.01)$ than $p a-$ tients rehabilitated with partial dentures. There was no difference for criteria of comfort, esthetics, phonetics, palate alteration, and pain $(p>0.05)$. Conclusions: the type of prosthesis may influence the rehabilitation prognosis. Moreover, patients rehabilitated with complete dentures reported more perception of improvement in quality of life after rehabilitation.

Keywords: Dental prosthesis. Patient satisfaction. Quality of life.

\section{Referências}

1. SBBrasil 2010 - Pesquisa nacional de saúde bucal: proposta de projeto técnico para consulta pública [online]. Brasília, DF; 2009 [citado 2012 Maio 13]. Disponível em URL: http:// portal.saude.gov.br/portal/arquivos/pdf/pesquisa_nacional_ bucal_0609.pdf.

2. Brasil. Ministério da Saúde. Secretaria de Atenção à Saúde. Departamento de Atenção Básica. Coordenação Nacional de Saúde Bucal. Projeto SB Brasil 2003: condições de saúde bucal da população brasileira 2002-2003. Resultados Principais [online]. Brasília, DF; 2004 [citado 2012 Maio 13]. Disponível em URL: http://dab.saude.gov.br/CNSB/vigilancia. php.

3. Silva DR, Magalhães RF. Significados das perdas dentárias e da reabilitação oral, com o auxílio de prótese parcial removível, para os pacientes atendidos nas clínicas do curso de Odontologia da UNIVALI [Trabalho de Conclusão de Curso]. Itajaí: Universidade do Vale do Itajaí; 2007.

4. Silva ME, Magalhães CS, Ferreira EF. Perda dentária e expectativa da reposição protética: estudo qualitativo. Cienc Saúde Colet 2010; 15(3):813-20.

5. Vargas AMD, Paixão HH. Perda dentária e seu significado na qualidade de via de adultos usuários de serviço público de saúde bucal do Centro de Boa Vista, em Belo Horizonte. Cien Saúde Colet 2005; 10(4):1015-24.

6. Colussi CF, Freitas SFT. Aspectos epidemiológicos da saúde bucal do idoso no Brasil. Cad Saúde Pública 2002; 18(5):1313-20 
7. Ferreira AAA, Piuvezam G, Werner CWA, Alves MSCF. A dor e a perda dentária: representações sociais do cuidado à saúde bucal. Cien Saúde Colet 2006; 11(1):211-8.

8. Barbieri $\mathrm{CH}$, Rapoport A. Avaliação da qualidade de vida dos pacientes reabilitados com próteses implanto-muco-suportadas versus próteses totais convencionais. Rev Bras Cir Cabeça Pescoço 2009; 38(2):84-7.

9. Veyrune JL, Tubert-Jeannin S, Dutheil C, Riordan PJ. Impact of new prostheses on the oral health related quality of life of edentulous patients. Gerodontology 2005; 22(1):3-9.

10. Duarte LR, Rabelo VA, Bonecker M, Castro 1, Melo G, Cavalcanti VA. Avaliação da qualidade de vida em pacientes edêntulos totais reabilitados com implantes zigomáticos. Implantnews 2010; 7(3a-PBA):183-90.

11. Feine JS, Dufresne E, Boudrias P, Lund JP. Outcome assessment of implant-supported prostheses. J Prosthet Dent 1998; 79(5):575-9.

12. Pocztaruk RL, Frasca LC, Rivaldo EG, Mattia PR, Vidal RA, Fernandes E, et al. Satisfaction level and masticatory capacity in edentulous patients with conventional dentures and implant-retained overdentures. Braz J Oral Sci 2006; $5(19): 1232-8$.

13. Souza RF, Patrocínio L, Pero AC, Marra J, Compagnoni MA. Reliability and validation of a Brazilian version of the Oral Health Impact Profile for assessing edentulous subjects. J Oral Rehabil 2007; 34(11):821-6.

14. Siqueira AF, Prado CJ, Carvalho MP, Borges TF, Mendes FA, Neves FD, et al. Habilidade mastigatória, qualidade de vida e satisfação em portadores de overdenture e prótese parcial removível mandibular [online]. [citado em 2012 Maio 21]. Disponível em URL: www.seer.ufu.br/index.php/ horizontecientifico/article/download/6354/6582.

15. Fujimori T, Hirano S, Hayakawa I. Effects of a denture adhesive on masticatory functions for complete denture wearers - consideration for the condition of denture bearing tissues. J Med Dent Sci 2002; 49(4):151-6.

16. Allen F, Locker D. A modified short version of the oral health impact profile for assessing health-related quality of life in edentulous adults. Int J Prosthodont 2002; 15 (5):446-50.

17. Gonçalves JR, Wassall T, Vieira S, Ramalho AS, Flório FM. Impactos da saúde bucal sobre a qualidade de vida entre homens e mulheres. RGO 2004; 52(4):240-2.

18. Miotto MHMB, Barcelos LA, Velten DB. Avaliação do impacto na qualidade de vida causado por problemas bucais na população adulta e idosa em município da Região Sudeste. Ciênc Saúde Colet 2012; 17(2):397-406.

19. Slade G, Spencer AJ. Social impact of oral confort among older adults. Aust Dent J 1994; 39(6):358-64.

20. Darvell BW, Clark RK. The physical mechanisms of complete denture retention. Br Dent J. 2000; 189(5):248-52.

21. Costa APS, Machado FCA, Pereira ALBP, Carreiro AFP, Ferreira MAF. Qualidade técnica e satisfação relacionadas às próteses totais. Ciênc Saúde Colet 2013; 18(2):453-60.

22. Costa APS, Machado FLA, Pereira ALBP, Carreiro AFP, Ferreira MAF. Qualidade técnica e satisfação relacionada às próteses totais. Ciênc Saúde Colet 2013; 18(2):453-60.

23. Silva EF, Sousa ML. Autopercepção da saúde bucal e satisfação com a vida em mulheres idosas usuárias de prótese total. Rev Odontol Univ Cidade São Paulo 2006; 18(1):61-5.

\section{Endereço para correspondência:}

Jessica Mie Ferreira Koyama Takahashi Escola Superior de Ciências da Saúde - UEA Secretaria do curso de Odontologia Avenida Carvalho Leal 1777, Cachoeirinha 69065-001 Manaus, Amazonas

Recebido: 09/06/2013. Aceito: 10/09/2013 